\title{
STAR FORMATION HISTORIES OF LOCAL GROUP DWARF GALAXIES
}

\author{
E.K. GREBEL \\ Astronomisches Institut Universität Würzburg \\ Am Hubland, D-97074 Würzburg, Germany
}

\section{Introduction}

The Local Group contains 37 currently known or probable member galaxies within a radius of 1.8 Mpc (Grebel 1997a). 31 of these galaxies are dwarf galaxies with $M_{B}>-17$ mag. The most frequent galaxy types are low-mass, gas-poor dwarf spheroidal (dSph) galaxies, which are dominated by old and intermediate-age populations, and more massive, gas-rich dwarf irregular (dIrr) galaxies with recent star formation. DSph galaxies have been found mostly in close vicinity to the large spiral galaxies in the Local Group, while dIrr galaxies tend to be more distant and isolated.

Knowledge of the star formation histories of these nearby galaxies will help us to evaluate the influence of galaxy mass, metallicity, and location with respect to other galaxies on galaxy evolution, to explore different star formation processes, and to understand distant, unresolved galaxies.

\section{Methods to study star formation histories}

Due to their proximity Local Group galaxies can be resolved into individual stars, which allows us to study their star formation histories in great detail. A variety of mostly photometric methods is employed ranging from studies of the stellar content to synthetic colour-magnitude diagrams. Poorly known and variable reddening, distance uncertainties, crowding, and lack of main-sequence photometry for older populations often make the derivation of star formation histories difficult. Spectroscopy and radio studies provide additional information on spectral types, abundances, and kinematics.

Proper motions are essential for determining orbits and constraining past and future interactions, but are extremely difficult to obtain due to the required large epoch differences.

\section{Spatial variations in star formation}

Stellar population studies in nearby galaxies often concentrate on one single field within a galaxy, which is then taken to be representative of the entire galaxy. However, even the lowest-mass galaxies known, the dSphs, turn out to show spatial variations of star formation rates and times.

For example, in the center of the predominantly intermediate-age dSph Fornax star formation lasted at least 5 Gyr with considerable enrichment (Beauchamp et al. 1995, Grebel 1997b), while only $570 \mathrm{pc}$ north of the center star formation proceeded for only about 2 Gyr with hardly any change in metallicity (Grebel 1995, 1997a). A comprehensive picture of the star formation history requires large-area coverage with deep photometry.

\section{Compiling star formation histories of all Local Group dwarves}

Using own and all available literature data of stellar population studies in Local Group galaxies, I derived their star formation histories and visualized them through population boxes (Grebel 1997a). A population box (Hodge 1989) is a 3-D representation of the star formation history of a galaxy showing the evolution of star formation rate and metallicity with time. Care has to be taken since input data are quite heterogeneous and differ in data quality, methods, and models used. 
In summary, I find that all Local Group dwarf galaxies

- contain old (>10 Gyr) and intermediate-age (1-10 Gyr) populations irrespective of morphological type,

- vary widely in star formation histories, metallicities, fractions, and ages of their subpopulations even within the same morphological type, and

- show complex star formation histories even within their older populations, and spatial variations in age and metallicity.

Objectives for future studies include accurate distances, accurate main-sequence turnoff ages, large-area coverage, and proper motions. The results will help us to understand star formation mechanisms, the role of interactions, and the formation history of the Local Group as a whole, and can then be extrapolated to distant galaxies.

\section{References}

Beauchamp, D., Hardy, E., Suntzeff, N.B., Zinn, R. (1995), AJ, 109, pp. 1628-1633

Grebel, E.K. (1995), PhD Thesis, Bonn University

Grebel, E.K. (1997a), Ludwig-Biermann-Award Lecture, Reviews in Modern Astronomy, Vol. 10, pp. 29 60

Grebel, E.K. (1997b), Invited review at the AAS topical session on "Old Stellar Populations Beyond the Milky Way", $B A A S$, Vol. 29, p. 825

Hodge, P.W. (1989), ARA\&A, Vol. 27, pp. 139-159 